\title{
Endophysics: The World As An Interface
}

\author{
Otto E. Rössler, World Scientific Publishing Co. Pte. Ltd, Singapore, New Jersey, London, \\ Hong Kong, 1998, 204 Pages, Index Included
}

\author{
ICHIRO TSUDA $^{\mathrm{a}, *}$ and TAKASHI IKEGAMI ${ }^{\mathrm{b}}$ \\ ${ }^{\mathrm{a}}$ Applied Mathematics and Complex Systems Research Group, Graduate School of Science, Department of Mathematics, Hokkaido University, Sapporo

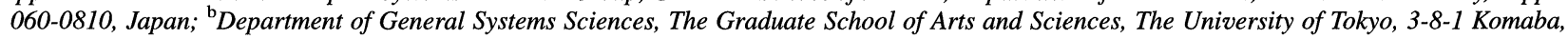 \\ Meguro-ku, Tokyo 153-8902, Japan
}

(Received 1 November 2001)

The ultimate purpose of science is to reconstruct a world by clarifying a modality of motion and structure. It is also possible to express virtual worlds and to hopefully synthesize them via mathematics and computers. The latter makes it possible for the former to appear in the world. By this act, scientists try to answer the question, "what is reality?".

There are two main perspectives through this enterprise. One is toward the outer world, and the other is toward the inner world. The former is related to what has been taken to be conventional scientific discipline. A typical case is seen in conventional physics, and is called "exophysics" in this book. The latter enables us to be conscious of selfreferential situations, that is, the situation of including an observer in a theory.

Otto E. Rössler took a big step towards the challenge of the latter direction about 20 years ago. He used the word "endophysics", after Finkelstein, to emphasize a viewpoint from within. Rössler's philosophical background of chaos theory and his own finding of the so-called "Rössler attractor" form the basis of his thoughts on endophysics, and going beyond conventional chaos theory, his project on endophysics includes the problems of individuality and selfness, which have been neglected in exophysics. This book, "Endophysics", is an edited book of Otto Rössler's scientific papers on this theme, organized by Peter Weibel, who is a most suitable person for this task.

In exophysics, reality simply obeys naive existentialism, that is, the existence of molecules and mechanics forms the reality of the surrounding world. In endophysics, reality is attributed to the interface between an observer and the rest of the world. This unique reality is comparable with the Kantian "fake reality" and/or psychoanalysis, but was able to be characterized in more scientific way by Rössler: namely, as an objective reality of a subjective type. Here, for instance, Rössler introduced a new concept of second causality as assignment conditions that are the third ingredient that makes it possible for any motion to appear endophysically in addition to the initial conditions and laws in the Newtonian dynamics. Rössler stresses throughout this book that reality is observer-state-relative that naturally leads to a notion of interface or "cut". This awareness of cut is extremely important, since assignment conditions are necessary in the internal situation that any observed state should be determined relative to the observer's state at the interface.

Rössler says that in the history of physics, such interface-affecting causation has been taken seriously by Boscovich, Einstein, Bohr and Everett. Other examples may found in thermodynamics. Rössler has attempted to reconstruct a world from a microscopic level, that is at a level of quantum mechanics with a relativity theory-like concept. Thus, his aim is to relate a microscopic nonlocality to "explicit observer", that is, the brain which appears to work at a macroscopic level.

Countable macroobjects may not be countable in a microscopic level. Thus, indistinguishability becomes essential in endophysics. It seems to us that an origin of individuality can be derived using this line, because how to prescribe the situation of being distinguishable is an essence of endophysics. Otto Rössler is likened to Niels Bohr as being a man who sees through the truth in nature, and can even predict new phenomena with a philosophical way of thinking, or even with enlightenment. Like Bohr's complementarity principle, Rössler tries to construct a firm guiding principle that governs our sense of reality.

*Corresponding author. E-mail: tsuda@math.sci.hokudai.ac.jp 
Consistently, he emphasizes how our world moves relative to the observers, but at the same time, he insists on how exact and mechanically manipulative the world is. That's why Rössler refers to virtual reality and quantum mechanics many times in this book, and the attitude is very different from mere relativism or simple realism.

His belief in such a new principle of endophysics seems to rely heavily on his encounter with chaos, that effectively introduced the notion of interface and information into science.

Whether or not an internal observer can go outside the world and can acquire the same objectivity that an external observer can obtain, seems to be an important problem. Nanotechnology showed this possibility by providing a way of "controlling" atoms. Is there any other such tool, especially at the macroscopic level? The idea of the digital computer was born when Alan Turing externalized his thoughts processes as a manual calculation, and hence, the present digital computer can be viewed as one side of the brain's function. What is missing in the construction of Turing machine is the externalization of subjectivity. Interestingly, Alan Turing argues this aspect of subjectivity by proposing the so-called Turing test. What the Turing test implies is that an internal observer does not exist either internally or externally in a system, but appears at the interface between both.

Brain theories now try to internalize things that were once externalized, which is the same theme that endophysics has grappled with. Falsifying both classical AI and behaviourism approaches, endophysics provides a third approach.

We strongly recommend this book to all researchers who are interested in the promising directions of being taken by complex systems' research.
This book contains scientifically intensive papers, as well as some extensive papers. The descriptive style of the papers is unusual, but this may show a better amalgamation of scientific and philosophical thoughts. Researchers and artists who are engaged in computer-aided interactive art will also be enthusiastic about the significance of endophysics, and of the way of constructing interfaces. For them, Peter Weibel's guide is recommended as an introduction. We would like to emphasize that all biologists, especially, brain scientists and evolutionary biologists, should take seriously the discipline of endophysics, since in these fields, it is inevitable to ask "what is reality for 'us', and what is reality for 'object animals' in the laboratory experiment?" Endophysics tells us that reality only exists at interfaces. By perturbing the interface slightly, we can have different senses of reality. This is what we have learned from Maxwell's demon or Bohr's cut.

Therefore, we need to construct a theory for interfaces where micro demons can produce macro strangeness that is related to mind and consciousness.

Such an interface is vulnerable, and hence endophysics inevitably includes the concept of "vulnerability".

Reflecting on the origin of scientific discipline, we know that vulnerability appears as a characteristic of science, but this concept seems to have been eliminated in modern scientific disciplines. What can we say to various social problems which we are now facing in the world? Perhaps, we will not be able to solve any problem merely with generosity, but maybe able to by allowing vulnerability.

"Endophysics" is a gift to the world of the 21st century by one outstanding scientist. 


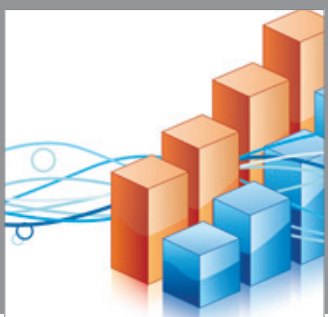

Advances in

Operations Research

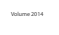

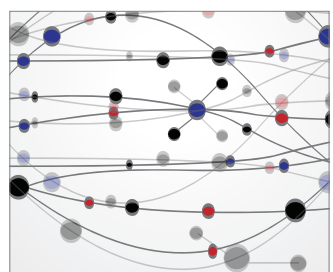

\section{The Scientific} World Journal
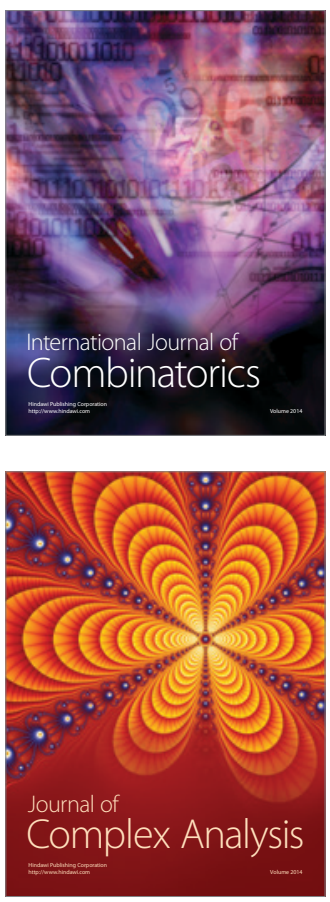

International Journal of

Mathematics and

Mathematical

Sciences
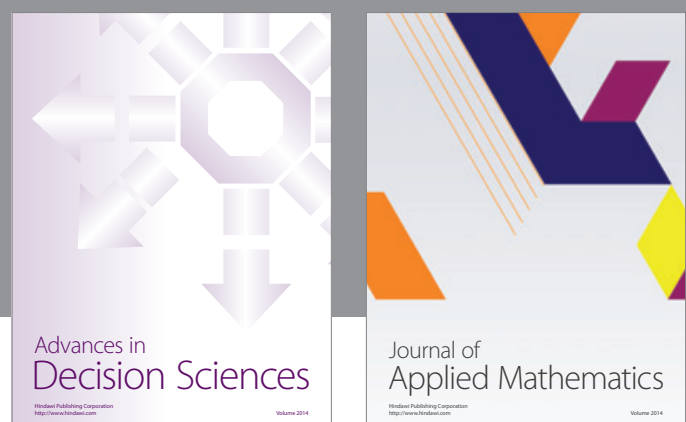

Journal of

Applied Mathematics
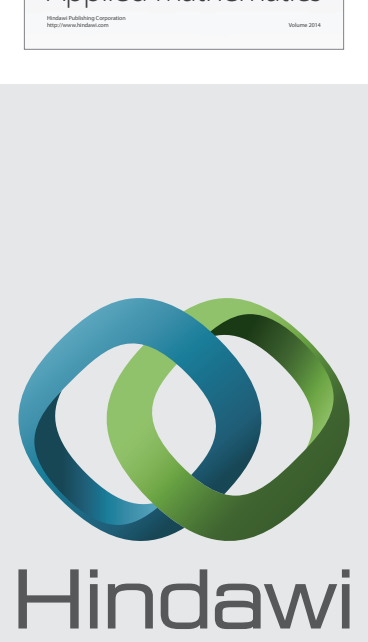

Submit your manuscripts at http://www.hindawi.com
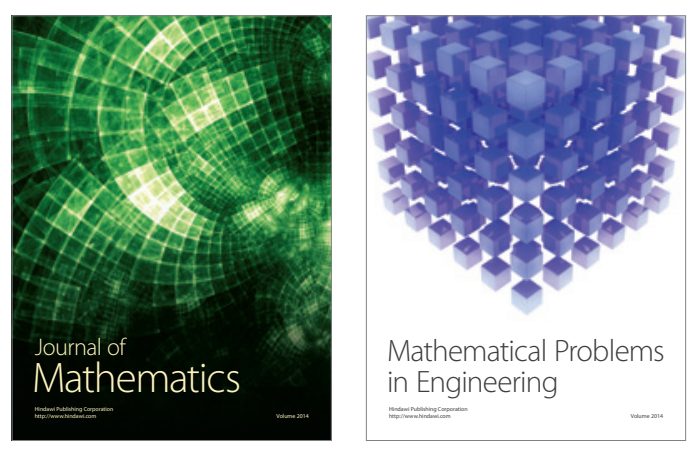

Mathematical Problems in Engineering
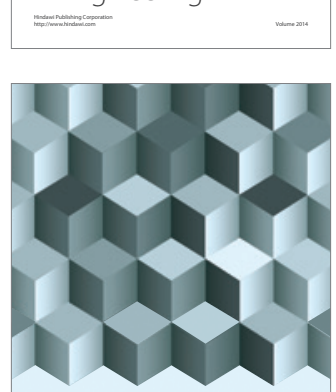

Journal of

Function Spaces
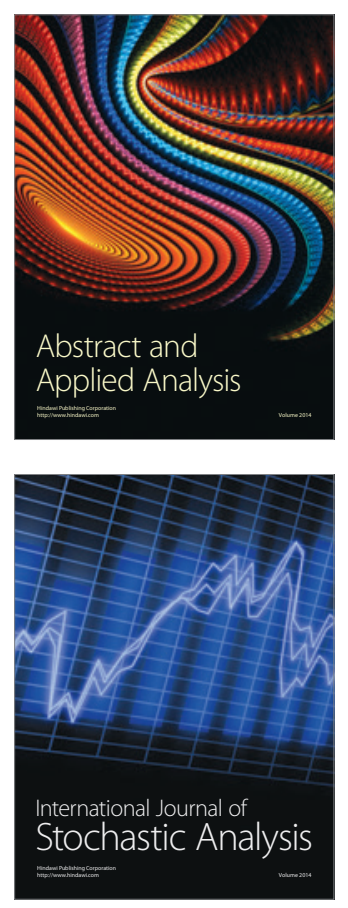

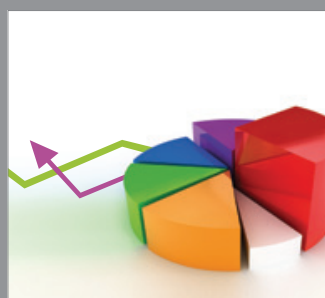

ournal of

Probability and Statistics

Promensencen
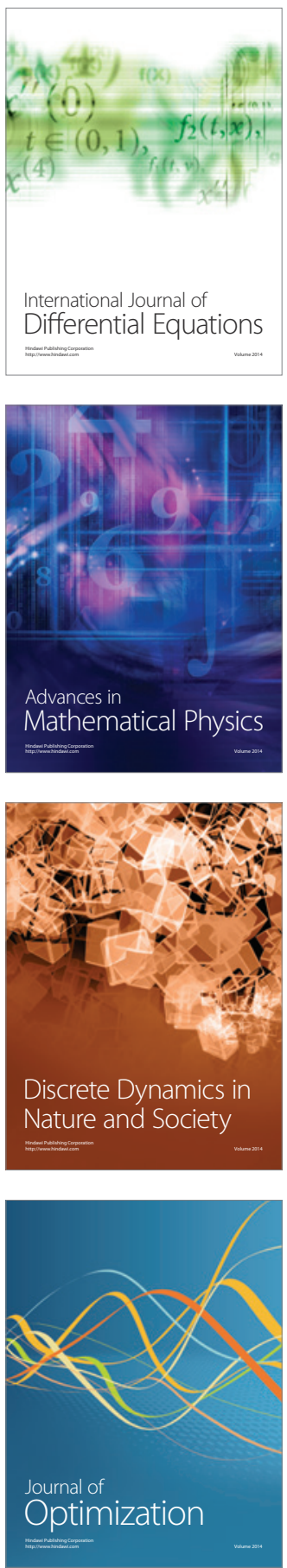